\title{
The ZARADEMP Project on the incidence, prevalence and risk factors of dementia (and depression) in the elderly community: II. Methods and first results
}

\author{
Antonio Lobo, MD* \\ Pedro Saz, MD* \\ Guillermo Marcos, MD** \\ José-Luis Día, MD*** \\ Concepción De-la-Cámara, MD* \\ Tirso Ventura, MD*** \\ José Angel Montañés, MD* \\ Antonio Lobo-Escolar, MD* \\ Sergio Aznar, MD* \\ and the ZARADEMP Workgroup
}

* Servicio de Psiquiatría and Departamento de Medicina y Psiquiatría, Facultad de Medicina of the Universidad de Zaragoza and Hospital Clínico Universitario;

** Departamento de Medicina Preventiva y Salud Pública, Facultad de Medicina of the Universidad de Zaragoza and Hospital Clínico Universitario;

*** Servicio de Psiquiatría, Hospital Universitario "Miguel Servet". Zaragoza

SPAIN

ABSTRACT - Background: There is a pressing need to identify environmental risk factors of dementia and depression in the elderly.

Aims: To describe the methods and first results of the ZARADEMP Project, the first psychiatric study to document risk factors in incident cases of dementia (and depression) in a Southern European elderly population.

Method: A three-wave (baseline and two follow-up waves) epidemiological study to identify incident cases of dementia (and depression) was completed in a representative sample population. Valid Spanish versions of case-finding instruments, including the Geriatric Mental State (GMS) were administered by standardized researchers. The incident cases, diagnosed by a panel of psychiatrists using DSM-IV criteria, will be included in a case-control study. 
Results: Response rate was acceptable (63.6\% in Wave I, 87'2\% in Wave II and 85 ' $8 \%$ in Wave III), and 4,803 individuals completed Wave I. Diagnostic agreement was achieved in $91.4 \%$ of probable cases presented to the panel, and a total of 146 incident cases of dementia were identified.

Conclusions: We feel confident that modern epidemiological requirements have been fulfilled and the statistical power in the calculations of risk will be acceptable.

\section{Introduction}

The aims and context of the ZARADEMP Project are described in the previous paper (Lobo et al. 2005, this issue). In summary, in a context of insufficient and, at times, contradictory findings in the epidemiology of dementia and depression in the elderly population, the main objective of the Project was to document the association between hypothesized risk factors and incident cases of dementia and its most frequent subtypes, in particular dementia of the Alzheimer's type (DAT). The ZARADEMP Project is also intended to study the stability of prevalence rates of dementia and depression in the same geographical area; the incidence rates and risk factors; the outcome of so called "mild cognitive deficit"; and to compare the findings in Zaragoza with results in other European enquiries also incorporated in the EURODEM and EURODEP Studies (Launer et al. 1992, Copeland et al. 1999). This report describes the methods and first results of the Project.

\section{Methods}

\section{Study city}

The site of the study was the historical city of Zaragoza, Spain. It is the capital of the historical kingdom of Aragón. It has been traditionally an agricultural area, although modern industrialization over the last 30 years has produced important migratory movements. One of the most important of these is the concentration in the city of Zaragoza of 622,371 inhabitants (fifth city in the country) or $51 \%$ the population of Aragón. The population aged 55 or more years in the city is 157,787 , or $43 \%$ the population of Aragón in this age group.

\section{Strategy of the study}

This is an epidemiological study with three waves (Figure 1). The previous Zaragoza Study (which we now call ZARADEMP “ 0 ") (Lobo et al. 1995) was the background investigation, and has influenced decisions about sampling, instruments and methods. Wave I (ZARADEMP I) is a baseline, cross-sectional study, intended to identify a cohort of individuals without dementia, as well as the prevalence and distribution of the hypothesized risk factors for dementia. It was also intended to document the prevalence and distribution of dementia, depression and psychiatric morbidity, for comparative purposes. The field work for this baseline study was to be completed by standardized lay interviewers, supervised by research psychiatrists.

Waves II and III (or ZARADEMP II and III) were designed to follow-up (two and four years later, respectively) the cohort of nondemented individuals, so that sufficient number of incident cases of dementia to test the main hypotheses would be identified at the end of the study. A two-phase screening (lay 


\section{Background}

"Zaragoza Study": ZARADEMP ${ }^{+} 0$ "

Prevalence of dementia and depression

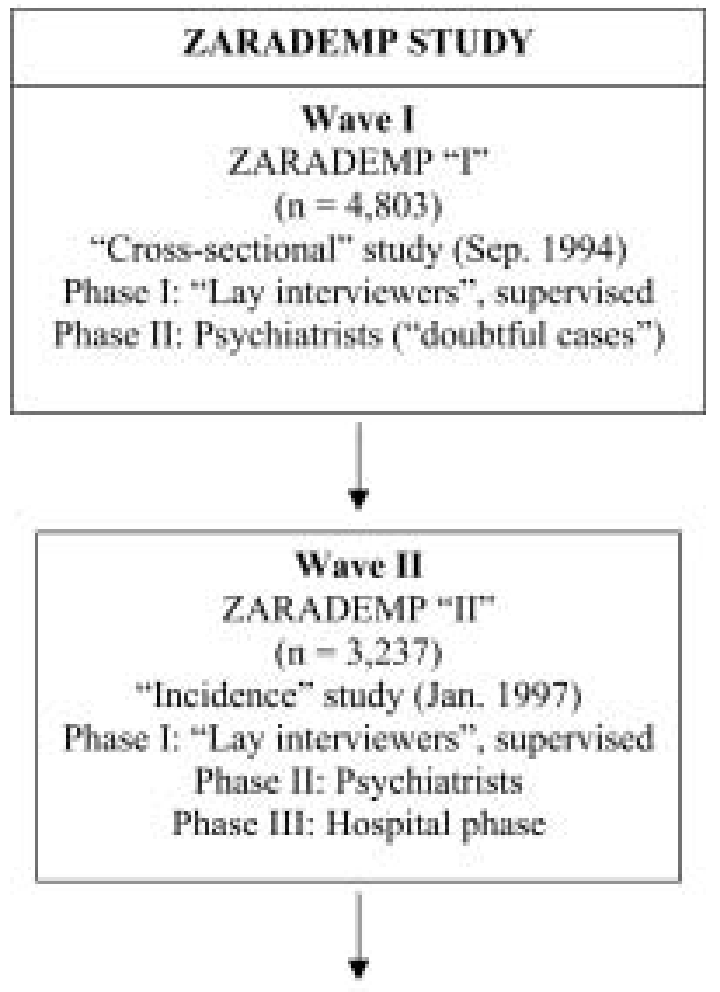

$$
\begin{gathered}
\text { Wave III } \\
\text { ZARADEMP "TII" } \\
(\mathrm{n}-2,403) \\
\text { "Incidence" study (May 1999) }
\end{gathered}
$$

Phase I: "Lay interviewers", supervised

Phase II: Psychiatrists

Phase III: Hospital phase 
interviewers and psychiatrists) would be completed in both waves. The elderly identified as probable incident cases of dementia would be invited to complete a third, hospital phase of assessment. A final diagnosis of dementia was to be approved by a panel of psychiatrists. In the principal stage of this investigation, a case-control study would also be completed in the incident cases of dementia identified in Waves II and III.

\section{Sampling technique and sampling size as a function of type I and type II errors}

It was decided to work with a random sample from the census list in the city of Zaragoza (1991) of both, men and women aged 55 years or more, stratified by sex and age (5-year age categories). The following considerations guided the design:

a) Incidence data reported in the international literature were rather provisional and rates quite variable. However, in view of data from ZARADEMP "0" (Lobo et al. 1997), the expected global incidence rate was 1 case per 100 persons/year;

b) Case-control studies reported until that time used hospital or prevalent cases, and "odds ratios" differed in relation to different factors analysed;

and c) The distribution of potential risk factors selected also varied considerably in the controls in different studies.

Since the main objective of this project was to obtain reliable measures of causeeffect association, sample size for the casecontrol study was calculated to allow the detection of relative risk $(\mathrm{RR})=3$, with a $95 \%$ confidence level and a power of $80 \%$ or more. In view of the literature examined, a mean frecuency of $5 \%$ of exposition to potential risk factors was considered, taking the example of traumatic brain injury. Higher frequencies have been reported for factors such as significant life events or family history of dementia, but also lower frequencies for factors such as Down's syndrome or Parkinson's disease.

One hundred and seventy nine incident cases of dementia were considered to be adequate to have good statistical power in the calculations of risk (Schlesselman and Stolley 1982). To obtain this number of cases, and taking into consideration the expected global incidence rate and logistics, including resources in the research group and timing of the study, to coincide with other projects incorporated in the EURODEM studies, a 5year follow-up of approximately 3,580 nondemented subjects was considered to be adequate. In calculating the initial sample size, this project is also supported on the following data from Zarademp " 0 ”): a) an expected $5.5 \%$ prevalence of global dementia; b) the high negative predictive value of the screening instruments (crucial in Wave I, when we were selecting non-demented individuals for the follow-up study), such as the Spanish versions of both, Mini Mental Status Examination (MMSE, Folstein et al. 1975) (97.8\%) and Geriatric Mental State (GMS, Copeland et al. 1987) (97.8\%) (98.3\%); c) and nonresponse rate. All the previous data are known by age strata. Similarly, estimations could be done about the distribution by age and sex in the population; about the expected annual migration (average) in the years 19861991; and about annual mortality rates, specific for age and sex.

According to this, and taking into consideration the less optimistic expectations, the initial sampling size, calculated to estimate the frequency of potential risk factors with a $2 \%$ error, was 10,916 individuals. It was corrected to 9,739 individuals after the first 
year of field study, in view of the preliminary data related to prevalence of dementia and cognitive deterioration, mortality, nonresponse, migration declared in the census and migration detected by interviewers.

\section{Instruments}

Several international instruments previously standardized in Spain by the same research group were incorporated in the ZARADEMP Interview. For the purpose of this report, the following will be described:

\section{Examen Cognoscitivo Mini-Mental} (ECMM) (Lobo et al. 1980), the official Spanish version of the MMSE, a reliable and valid indicator of cognitive disturbance and probably the test of cognitive function in most widespread use. Modifications were introduced in this official version, to minimize potential bias due to a low educational background. Efficiency coefficients and population norms very similar to the ones reported by Folstein et al in the USA have been found in Zaragoza (Lobo et al. 2002).

Geriatric Mental State (GMS), a well known semistructured standardized clinical interview for assessing the mental state of elderly persons. The $G M S-B$, a shortened community version that may be used by lay interviewers has been selected in this study. It covers the important symptom areas for disorders likely to be encountered in the community and the computerized program $A G E$ $C A T$ can be applied to it (Copeland et al. 1987). This interview is also a syndrome case finding instrument: the $G M S-B$ "threshold global score" discriminates between "noncases"and "cases". The reliability and validity of the backtranslated, Spanish version of the $G M S-B$ has been previously reported in hospital samples and in the general population both, for lay interviewers and for psychiatrists (Lobo et al. 1988, Saz 1991).
Automated Geriatric Examination for Computer Assisted Taxonomy (AGECAT) (Copeland et al. 1986), a set of computer programs which analyse GMS data. Stage I of the AGECAT groups the items of the GMS into symptom components, gathered under eight diagnostic "clusters" (or "syndromes"). In Stage II, all levels on each cluster are then compared across clusters and the subject emerges with a main diagnosis and a subsidiary or alternative diagnosis if appropriate. In the organic brain syndromes it has been shown to correspond to diagnoses of dementia made according to the rules of DSM-III-R (American Psychiatric Association 1987). Data about the validity of the Spanish version have also been reported (Lobo et al. 1990).

History and Aetiology Schedule (HAS), a standardized method of collecting history and aetiology data from an informant, or directly from the respondent when he is judged to be reliable. It concentrates on those features expected to be relevant to psychiatric diagnosis in older people, and is crucial to complete the GMS and facilitate a diagnostic process such as the one done in the present study with the DSM-IV system (Copeland et al. 1992, American Psychiatric Association 1994). A computerized diagnostic method applicable to the standardized psychiatric schedule has been developed, the $H A S-A G E C A T$, and we have reported good agreement (kappa $=0,76)$ with the interviewing psychiatrist when using the Spanish version (Dewey et al. 1992).

Disability Scales. The scale for Instrumental Activities of Daily Living (IADL, Lawton \& Brody 1969) is a widely used instrument, a reliable and valid indicator of disturbance in both, basic and instrumental activities of daily living. It may be administered by lay interviewers, the information coming from both, patients and caregivers. A 
series of studies completed in Spain have documented in clinical samples efficiency coefficients very similar to the ones reported by the original authors (Tárraga 1995). The Katz Index is also a well known, reliable and valid instrument, intended to assess difficulties in basic activities of daily living (BADL) in the elderly (Katz et al. 1963). Studies completed in Spain have also confirmed the validity of the scale (Álvarez et al. 1992). In this study, the interviewers were instructed to score in both instruments only disability due to deterioration in cognitive function.

The Risk Factors Questionnaire used in this study was designed by the EURODEM Workgroup (Launer et al. 1992). The instrument may be used by trained lay interviewers, and is intended to include information related to the following potential risk factors of dementia, DAT and VD: history of medical diseases, including cardiovascular disease (hypertension, myocardial infarct, angina), cerebrovascular accident (CVA), traumatic brain injury, epilepsy, Down's syndrome, Parkinson's disease, diabetes mellitus, thyroid disease, abuse of alcohol or smoking; menopause; psychiatric history, in particular depression; tobacco and alcohol habits; use of medications, including psychopharmaca; and history of general health. Each item in the interview has been operationally defined, according to previously agreed EURODEM criteria.

The ZARADEMP Interview also incorporates a section on physical examination, including the checking of blood pressure following World Health Organization standards (WHO 1993), height and weight of the individual.

\section{Procedure}

In all three waves of this research, the elderly were initially informed about the study through an individually signed letter. Then, an appointment was done by telephone call when possible or else, the home address was visited. Up to three home visits were done before it was considered to have an address problem or that the individual was not found. In a small number of subjects who objected to being approached, the senior researchers contacted the individuals personally. If the individual happened to be institutionalized, he/she was interviewed in the institution. Written informed consent was obtained from each participating individual (a relative when he/she was unable to understand the terms of the agreement), according to the current Spanish laws. The family telephone and address were registered, as well as relevant medical and psychiatric information given by the family that might be useful in cases of refusal or loses in the follow-up waves.

\section{Case finding procedures and study design}

\author{
Wave I: "Baseline", "cross-sectional" \\ study
}

An epidemiological screening design was used in this wave, the field study beginning in September 1994. Lay interviewers administered the ZARADEMP Interview, with all the incorporated instruments. The blood pressure, height and weight of each individual were also checked and recorded routinely in this phase of the study. Medical reports, which are commonly kept at home by Spanish patients and may include laboratory data, were used in some cases to help in the diagnostic process.

The interviews lasted 25-90 minutes. The individuals were nominated as "probable cases" on the basis of GMS threshold "global" scores and/or ECMM standard cut-off points, which we previously reported to have good validity coefficients (Lobo et al. 1980, 
Lobo et al. 1988). The threshold points here were decided on the bases of adequate negative predictive value. However, the data on each elderly were thoroughly reviewed by the research psychiatrists supervising individually the lay interviewers. In the final step of Wave I, the psychiatrists recorded a diagnosis of "dementia", "depression" or "others" in the nominated "cases". For a diagnosis of dementia, documented deterioration in activities of daily living due to cognitive deterioration was required. In the doubtful cases, the research psychiatrists repeated the examination in the elderly home.

"Subcases" of "dementia" were also nominated on the basis of borderline scores on the same instruments. Some of these "subcases" might be incipient cases of dementia, and therefore might contaminate both, the incidence study and the study of risk factors in incident cases. Therefore, they were eliminated for Waves II and III. However, they will be included in a different sub-study, a followup to document the natural course of mild cognitive disturbance and cognitive disturbance in non-demented individuals.

\section{Waves II and III: Follow-up study}

The elderly considered to be neither "cases", nor "sub-cases" of dementia or cognitive deterioration in the previous wave are the cohort for the "follow-up" studies, Wave II or ZARADEMP II and Wave III or ZARADEMP III, to be completed with intervals of (approximately) two years, to document the incidence of dementia. Wave II started in January 1997, and Wave III in May 1999. Similar procedures will be used to document the incidence of depression. A two-phase epidemiological screening (Phases I and II), was designed in both follow-up waves.

In Phase I, lay interviewers administered the ZARADEMP Interview and nominated "probable cases" with the same procedure described in Wave I. However, the interviewers were instructed to be "sensitive", since the false positive cases could be corrected in the second phase. All "probable cases" of dementia were examined in Phase II, two months later and blind to the results of Phase $I$, by research psychiatrists standardized in the methods. All GMS "borderline noncases" were also examined, to correct for possible errors in the screening procedure and to minimize the risk of false negative cases. The period of two months between phases was decided in view of previous experience of the group, to minimize the possibility of false positive cases of dementia due to acute brain syndromes. The psychiatrists also used the ZARADEMP Interview and the incorporated instruments, including the Hachinski Scale (1975) to help in the differentiation between primary degenerative and vascular dementia. The elderly were examined at home, the exception being the institutionalized individuals, or individuals preferring to come for assessment to the out-patient department in the hospital. A neurological examination was performed and medical reports were used when available, to help in the diagnostic process. Outside informants were interviewed when the selected elderly was considered to be unreliable.

All probable "incident cases" and doubtful cases of dementia identified by the psychiatrists were then presented to a panel of psychiatrists, who examined all available documentation. For the diagnosis of "incident case" of dementia, and type of dementia, agreement of at least three out of four psychiatrists was considered necessary. While previous experience in ZARADEMP " 0 " suggested the reluctance of the elderly to come to the hospital for assessment, a Phase III or hospital phase was also programmed to confirm the diagnosis of dementia, and type of dementia, in as many patients as possible. 
The assessment protocol will be described in the following section.

\section{Diagnostic criteria}

At the end of Phase II, the psychiatrists diagnosed the identified "cases" of dementia according to DSM-IV criteria (American Psychiatric Association 1994). Both, project criteria (Lobo et al. 1988) and ICD-10 (World Health Organization 1992) criteria of dementia were also recorded, to eventually report comparative results. The ZARADEMP Interview used in this study generates enough information to diagnose according to all these criteria the main nosological categories found in the elderly community, specifically dementia (its most prevalent types, that is, DAT and VD) and depression (its various types). For the DSM-IV diagnosis of type of dementia a protocol was built, so that the requirements for each one of the items in the diagnostic manual were operationalized with information coming from the items in the ZARADEMP Interview. Our previous studies have supported the validity of the diagnosis of dementia done by research psychiatrist in the elderly homes (Saz et al. 1996).

For the diagnosis of dementia in the hospital phase, a neurological, dementia protocol used in previous studies of the group (Lobo et al. 1995) was completed in all cases referred. This protocol allows the classification of cases of DAT according to NINCDSADRDA criteria (McKahnn et al. 1984), and cases of VD according to NINDS-AIREN criteria (Roman et al. 1993).

\section{"Case-control" study of the incident cases of dementia}

The "incident cases" of both DAT and VD identified in the follow-up waves will be included in the "case-control" study. The controls will be randomly selected among individuals at risk, but having no dementia, matched with cases by age (up to two years difference) and gender. Since the information about potential risk factors incorporated in the ZARADEMP Interview was collected in the "baseline study", it includes data related to exposition both, in cases and in the general population. Consequently, it will be possible to test causal hypotheses with enough statistical power.

\section{Database elaboration and quality control}

A systematic control of the reliability of assessments was programmed and implemented every six months, in all three waves, to prevent the "reliability-decay". In case the decay was systematic, a re-standardization of interviewers was programmed, which included video techniques used in the initial training. Furthermore, a periodic and systematic control of interviewers' activity was programmed, and included the registration and analysis of response rate and reasons for refusal for each interviewer, to assure the homogeneity of data. The database design and quality control were done according to standard methods previously used by our research group, including the analysis of "out of rank" data and non-coherent information.

A preliminary report was completed at the end of every consecutive year and included descriptive statistical analysis performed with SPSS. In this way, it was possible to confirm that the prevalence, distribution and incidence of morbidity found in the population was coherent with the hypotheses formulated. Similarly, it was possible to verify in a systematic way that the distribution of potential risk factors assessed with the ZARADEMP Interview for both, dementia 
and depression were also coherent with the working hypotheses.

\section{Statistical procedures}

Statistical methods will vary depending on the particular study. Proportional contrast" statistics will be used to estimate simple measures, such as response and refusal rates. The prevalence rates (and confidence limits) of psychiatric disorder and of dementia, dementia subtypes and depression will be estimated from the stratified sample of the elderly responders to the clinical evaluation. The incidence rate of both dementia and depression will be documented in relation to variables studied and factors theoretically associated with them. In a second step, risks associated to exposition to different factors will be estimated, by calculating odds ratios and confidence intervals. Standard univariate analysis techniques common in epidemiology will be used, as well as logistic regression and multivariate analysis techniques. Survival analysis techniques will be used to study survival time variables, such as mortality. Standard statistical packages (SPSS 11.01 for Windows and EpiInfo 6.04 for MsDos) will also be used throughout the analysis.

\section{Ethical and legal issues}

The Helsinki convention principles have been maintained throughout the study, as well as the compromise of information to the elderly, written consent, privacy, confidentiality and security, according to Spanish Law 5/1992. The collected information was considered "sensitive data", and a "dissociation procedure" was implemented. Sealed envelopes with individual records, under the custody of the principal researcher, had to be opened previous to the programmed followup interviews in each elderly. Individual files will be destroyed after validation of the pertinent information by sampling, in the end step of the investigation.

\section{Connection with the EURODEM and EURODEP studies}

Data of the Zarademp Project will be eventually compared to data collected with similar methodology in both the EURODEM (Launer et al. 1992) and EURODEP Studies (Copeland et al. 1999). Preliminary results of this project have been reported in relation to the prevalence and incidence of dementia (Fratiglioni et al. 2000; Lobo et al. 2000). Particular emphasis will be placed in the comparison of risk factors for dementia, according to the original protocol (Launer et al. 1992).

\section{Results}

\section{Sample characteristics and response rate}

Of the initial, total sample $(\mathrm{n}=9,739)$, 4,803 individuals completed Wave I of the study. Refusal rate was $20.5 \%(\mathrm{n}=2,001)$. Table I shows the distribution by gender and age group at the time of interview. Table II shows other demographic characteristics of the sample: most the elderly were either currently married or widowed and the majority had a limited, primary education. After removal of cases and subcases of dementia for the follow-up ( $\mathrm{n}=742), 4,061$ individuals were approached for Wave II, which was completed by 3,237 individuals, the refusal rate being $9.9 \%(n=402)$. Three thousand one hundred and sixty individuals were approached for Wave III, which was completed by 2,403 individuals, the refusal rate being $9.3 \%(n=293)$. While the distribution 
of the elderly according to most demographic characteristics is similar in all three waves, the proportion of illiterate individuals in Wave $I(10.8 \%)$ is significantly higher than in both, Wave II $(7.5 \%)(\mathrm{z}=4.92, \mathrm{p}<$ $0.0001)$ and Wave III $(6.3 \%)(\mathrm{z}=6.16, \mathrm{p}<$ $0.0001)$. The differences between Waves II and III did not reach statistical significance) $(\mathrm{z}=1.70, \mathrm{p}=0.09)$ (Table II).

Table III summarizes reasons for nonresponse, by gender. In all three waves, the proportion of non-response due to refusals was significantly higher in women when compared to men: in Wave I, it was $43.7 \%$ vs $35.9 \%(\mathrm{z}=5.44, \mathrm{p}<0.0001)$; in Wave II, it was $54.6 \%$ vs $41.8 \%(\mathrm{z}=3.6, \mathrm{p}<0.0001)$; and in Wave III, it was $46.9 \%$ vs $29.6 \%$ ( $\mathrm{z}=$ $4.80, \mathrm{p}<0.0001)$.

In Wave I, 159 individuals were considered to be "doubtful cases" of dementia by lay interviewers, and 79 of them were considered to be cases by the research psychiatrist after examination in the elderly home. In Waves II and III, one hundred and seventy "probable" or "doubtful" incident cases of dementia were identified by the research psychiatrists following the described case finding procedure and were presented to the diagnostic panel. Agreement of the panel was achieved in 160 indi-

Table I

Sample characteristics of the elderly interviewed

\begin{tabular}{lrrrrrrrrr} 
& \multicolumn{2}{c}{ ZARADEMP I } & \multicolumn{3}{c}{ ZARADEMP II } & \multicolumn{3}{c}{ ZARADEMP III } \\
Age & Male & Female & Total & Male & Female & Total & Male & Female & Total \\
\hline $55-59$ & 77 & 88 & 165 & 0 & 0 & 0 & 0 & 0 & 0 \\
$60-64$ & 439 & 484 & 923 & 254 & 279 & 533 & 68 & 77 & 145 \\
$65-69$ & 391 & 474 & 865 & 333 & 382 & 715 & 272 & 295 & 567 \\
$70-74$ & 345 & 492 & 837 & 293 & 358 & 651 & 263 & 291 & 554 \\
$75-79$ & 245 & 372 & 617 & 218 & 317 & 535 & 196 & 292 & 488 \\
$80+$ & 535 & 861 & 1,396 & 350 & 453 & 803 & 262 & 387 & 649 \\
Total & 2,032 & 2,771 & 4,803 & 1,448 & 1,789 & 3,237 & 1,061 & 1,342 & 2,403 \\
\hline
\end{tabular}

Table II

Demographic characteristics of the sample

\begin{tabular}{|c|c|c|c|c|c|c|}
\hline \multirow[t]{2}{*}{ Characteristic } & \multicolumn{2}{|c|}{ ZARADEMP I } & \multicolumn{2}{|c|}{ ZARADEMP II } & \multicolumn{2}{|c|}{ ZARADEMP III } \\
\hline & $\mathrm{N}$ & $(\%)$ & $\mathrm{N}$ & $(\%)$ & $\mathrm{N}$ & $(\%)$ \\
\hline \multicolumn{7}{|l|}{ Marital status } \\
\hline Single & 389 & $(8.1)$ & 228 & $(7.0)$ & 185 & (7.7) \\
\hline Married /common law & 2,796 & $(58.2)$ & 2,013 & $(62.2)$ & 1,468 & $(61.1)$ \\
\hline Widowed & 1,494 & (31.1) & 908 & $(28.1)$ & 692 & $(28.8)$ \\
\hline Separated / divorced & 65 & $(1.4)$ & 50 & $(1.5)$ & 40 & $(1.7)$ \\
\hline Religious & 45 & $(0.9)$ & 31 & $(1.0)$ & 17 & $(0.7)$ \\
\hline Missing & 14 & $(0.3)$ & 7 & $(0.2)$ & 1 & $(0.0)$ \\
\hline TOTAL & 4,803 & & 3,237 & & 2,403 & \\
\hline \multicolumn{7}{|l|}{ Education level } \\
\hline Illiterate & 521 & $(10.8)$ & 243 & $(7.5)$ & 151 & $(6.3)$ \\
\hline Some primary & 1,729 & $(36.0)$ & 1,102 & $(34.0)$ & 795 & $(33.1)$ \\
\hline Primary & 1,778 & $(37.0)$ & 1,285 & (39.7) & 978 & (40.7) \\
\hline High school & 363 & $(7.5)$ & 297 & $(9.2)$ & 240 & $(10.0)$ \\
\hline Some college or higher & 354 & (7.3) & 288 & $(8.9)$ & 226 & $(9.4)$ \\
\hline Missing & 58 & $(1.2)$ & 22 & $(0.7)$ & 13 & $(0.5)$ \\
\hline TOTAL & 4,803 & & 3,237 & & 2,403 & \\
\hline
\end{tabular}


viduals (91.4\%), and 146 of them were considered to be cases of dementia. In this way, $81.6 \%$ of the 179 incident cases of dementia initially calculated to complete the case-control study with good statistical power were identified.

Table III

Reasons for non-response, by gender

\begin{tabular}{lcccccccccc} 
& \multicolumn{1}{c}{ Moved away } & \multicolumn{2}{c}{ Dead } & \multicolumn{2}{c}{ Untraced } & \multicolumn{2}{c}{ Refusals } & Total \\
& $\mathrm{N}$ & $(\%)$ & $\mathrm{N}$ & $(\%)$ & $\mathrm{N}$ & $(\%)$ & $\mathrm{N}$ & $(\%)$ & $\mathrm{N}$ \\
\hline ZARADEMP I* & & & & & & & & & & \\
$\quad$ Women & 220 & $(7.5)$ & 968 & $(32.8)$ & 473 & $(16.0)$ & 1,289 & $(43.7)$ & 2,950 \\
$\quad$ Men & 127 & $(6.4)$ & 874 & $(44.1)$ & 269 & $(13.6)$ & 712 & $(35.9)$ & 1,982 \\
$\quad$ Total & 347 & $(7.0)$ & 1,842 & $(37.3)$ & 742 & $(15.0)$ & 2,001 & $(40.6)$ & 4,932 \\
ZARADEMP II & & & & & & & & & \\
$\quad$ Women & 32 & $(7.2)$ & 124 & $(28.0)$ & 45 & $(10.1)$ & 242 & $(54.6)$ & 443 \\
$\quad$ Men & 33 & $(8.6)$ & 161 & $(42.0)$ & 29 & $(7.6)$ & 160 & $(41.8)$ & 383 \\
$\quad$ Total & 65 & $(7.9)$ & 285 & $(34.5)$ & 74 & $(9.0)$ & 402 & $(48.7)$ & 826 \\
ZARADEMP III & & & & & & & & & \\
$\quad$ Women & 36 & $(9.0)$ & 118 & $(29.6)$ & 58 & $(14.5)$ & 187 & $(46.9)$ & 399 \\
$\quad$ Men & 31 & $(8.7)$ & 174 & $(48.6)$ & 47 & $(13.1)$ & 106 & $(29.6)$ & 358 \\
$\quad$ Total & 67 & $(8.9)$ & 292 & $(38.6)$ & 105 & $(13.9)$ & 293 & $(38.7)$ & 757 \\
\hline
\end{tabular}

* Four interviews were rejected in quality control procedures.

\section{Discussion}

\section{The design of the study}

We feel confident that the design of the study follows contemporary methodological standards in epidemiological studies (Kaelber and Regier, 1995): a randomized and representative community sample from the census list was investigated, the elderly being interviewed in each wave in their place of residence, even in residential institutions; and the instruments used for screening and case ascertainment had been specifically developed for the elderly, were widely accepted in the international literature, and were previously standardized in our specific culture. In this way, potential bias due to the influence of low educational background is minimized. Furthermore, the sensitivity coefficients of the instruments used here (GMS "Organic" = 93.2, Lobo et al. 1990; $\mathrm{ECMM}=89.8$, Lobo et al. 2002) are considered adequate and comparable to coefficients reported in several studies in this field (Hofman et al. 1991).
The advantage of designing a project such as the present one following a previous investigation in the same setting should not be underestimated. On the bases of the previous study, we had data about the predictive values of the case finding instruments, so that we could decide the threshold points to be used. We also had data about both prevalence and incidence of dementia, as well as the distribution by dementia type in the general population; and about the expected response rate. While the mortality rate before interview in Wave I may seem very high, it had been predicted in this large population $(\mathrm{n}=$ 157,787 ) from Spanish annual mortality rates reports (1985-1987) (INE, 1992).

In fact, both mortality and migration rates were slightly lower than expected, so that we were able to correct the sampling size initially calculated as a function of a case-control design. We were able to identify $81.6 \%$ of the incident cases of dementia initially considered in the design. Therefore, we believe the statistical power in the calculations of risk 
will not be seriously affected. It might be argued that basing the sampling size on a RR $=3$ for risk factors seems over-optimistic. Some potential risk factors may have a lower $\mathrm{RR}$ and hence be undetectable with this sample size. However, increasing considerably the sampling size would seriously limit the efficiency in our particular study.

In view of the main objectives of this Project, we have relied on recruitment of large numbers of subjects. Logistical reasons prevent a more detailed scrutiny to document the progression from mild cognitive impairment (MCI) to dementia (Amieva et al. 2004). However, we have also planned future, consecutive waves following Wave III, which should provide abundant data on the conversion rate of MCI to dementia.

Another factor that may influence the data on the epidemiological rate of dementing conditions refers to the diagnostic process itself. We are well aware of the difficulties in diagnosing dementia in the elderly's domicile, and were able to complete a full diagnostic, hospital protocol in only a proportion of patients. However, standardized levels of illness and diagnostic criteria were used (Copeland et al. 1987). The impact of different case definitions on incidence rates of dementia appears limited if case definitions and case-finding procedures at baseline and follow-up are applied consistently (RiedelHeller et al. 2001). In fact, we have shown good diagnostic agreement between the psychiatrists doing the clinical interview in the elderly's home and the panel reviewing the data: this agreement occurred in $91.4 \%$ of individual "cases" and "sub-cases" presented. It is still possible that we have applied DSM-IV criteria of dementia differently from other researchers, since they are not fully operational. Such shortcomings should be considered when reporting the comparability of studies, although we have followed previous recommendations (Hofman et al. 1991), by translating the clinical data into specific operational criteria.

\section{Sample attrition and response rate}

Important uncertainties in epidemiological studies relate to sample attrition. Therefore, we did an special effort to minimize this risk, and a considerable proportion of the elderly had to be traced to temporary places of residence in their childrens' homes or, especially in summer periods, to their temporary rural homes. We believe that the response rate in our study, and in particular the refusal rate have not seriously biased the results. Response rate in our study, after removal of expected losses from the sample (the moved away and the dead) was $63.6 \%$ in Wave I, $87^{\prime} 2 \%$ in Wave II and 85 ' $8 \%$ in Wave III. The refusal rate was $20.5 \%$ in Wave $I, 9.9 \%$ in Wave II and $9.3 \%$ in Wave III. Classical epidemiological texts (MacMahon and Pugh, 1970) consider quite acceptable a response rate below $70 \%$ in large studies such as the Framinghan study on vascular problems (Dawber et al. 1963). Similarly, our response rates are consistent with rates reported in epidemiological studies on dementia. Copeland et al (1987) reported a response rate of $72 \%$ in their one-stage investigation. In some twostage studies, authors such as Sulkawa et al (1985) in Finland reported a good cumulative rate of $87 \%$, but Folstein et al (1991) had a cumulative response rate of $52 \%$ and Dartigues et al (1991) had a response rate of $69.8 \%$ in the PAQUID Study.

\section{Non-responders and estimation of epidemiological rates of disorder}

It might be argued that non-responders would especially be at greater risk for a psychiatric disorder and, therefore, that we 
might have underestimated the burden of dementia (and depression) in this population. However, we could not confirm this hypothesis in ZARADEMP "O" (Lobo et al. 1995), which is the base for the present study and was completed in the same city. On the contrary, non-response due to refusals in the present report was consistently and significantly higher in women when compared to men. The losses among women should be considered to avoid underestimation of prevalence and incidence rates of depression, since depression has consistently been reported to be higher among women when compared to men both, in the international literature (Copeland et al. 1999) and in the city of Zaragoza (Lobo et al. 1995). Furthermore, the higher losses among women should also be discussed in relation to prevalence and incidence rates of DAT. While no significant differences by gender were observed in our city, a number of studies have reported higher epidemiological rates among women (Copeland et al. 1999, Andersen et al. 1999).

The significantly lower proportion of illiterate individuals found in the follow-up waves, when compared to the baseline wave, could be related to higher mortality in this educational group, a finding previously reported in some studies (Steenland et al. 2002). This hypothesis tends to be supported by the ad hoc analysis we completed. Non-response due to mortality was $37.3 \%$ (CI 95\%, 36.0 - 38.7) in Wave I; $34.5 \%$ (CI 95\%, 31.3 - 37.9) in Wave II; and $38.6 \%$ (CI 95\%, 35.1 - 42.2) in Wave $I I I$, the between waves differences being non significant (I vs II: $\mathrm{z}=1.5, \mathrm{p}=0.132$; II vs III: $\mathrm{z}=1.64, \mathrm{p}=0.101$ and I vs III: $\mathrm{z}$ $=0.65, \mathrm{p}=0.517)$. However, in coincidence with previous reports, the mortality was higher in the illiterate individuals $(51.5 \%, 48.4 \%$ and $40.0 \%$ respectively), and the differences were statistically significant in Waves I and II (not included in the interval coefficients). In view of the higher risk of dementia, DAT in particular, reported in individuals with limited education (Letenneur et al. 1999), the possibility that higher mortality among illiterate elderly may influence epidemiological rates in the ZARADEMP Project should be considered in the coming reports.

\section{Acknowledgements}

Supported by grants 94-1562, 97-1321E, 98-0103 and 01-0255 from the Fondo de Investigación Sanitaria, Ministerio de Sanidad, Madrid, Spain, by grant CICYT SAF93-0453 from the Dirección General de Investigación científica y Técnica, Secretaría General de Universidades, Madrid, and by grants from the Fundación CAI and Fundación Pfizer.

The following researchers from the ZARADEMP Workgroup are also authors in this paper: Quintanilla MA, Quetglas B, Zapata M, Martín A, Gurrea A, Sarasola A, Pelegrín C, Pascual A, Campos R, Alonso JP, Astier P, Morales F, Pascual LF, Salvador JA, Lacámara C, Laguardia P.

The authors acknowledge the contribution of the medical students and general practitioners, who participated in the field study as lay interviewers.

We also thank Robert G Robinson, at the Department of Psychiatry, Iowa University, USA, and John RM Copeland, at the Department of Psychiatry, University of Liverpool, United Kingdom, for their encouragement and helpful criticisms of the final draft of this article. 


\section{References}

Álvarez M, Alaiz T, Brun E et al. Capacidad funcional de pacientes mayores de 65 años, según el Índice de Katz. Fiabilidad del método. Aten Primaria 1992; 10: 812-816.

American Psychiatric Association. Diagnostic and statistical manual of mental disorders ( $3^{\text {rd }}$ Rev. Edition) (DSM IIII-R). Washington DC: American Psychiatric Press; 1987.

American Psychiatric Association. Diagnostic and statistical manual of mental disorders ( $4^{\text {th }}$ ed.) (DSM-IV). Washington, DC: American Psychiatric Press; 1994.

Amieva H, Letenneur L, Dartigues JF et al. Annual rate and predictors of conversion to dementia in subjects presenting mild cognitive impairment criteria defined according to a population-based study. Dement Geriatr Cogn Disord 2004; 18(1): 87-93.

Andersen K, Launer LJ, Dewey ME, et al. Gender differences in the incidence of AD and vascular dementia: The EURODEM Studies. EURODEM Incidence Research Group. Neurology 1999; 53 (9): 1992-1997.

Copeland JRM, Beekman ATF, Dewey ME et al. Depression in Europe: Geographical distribution among older people. Br J Psychiatry 1999; 174: 312-321.

Copeland JRM, Davidson IA, Dewey ME et al. Alzheimer's disease, other dementias, depression and pseudodementia: prevalence, incidence and three-year outcome in Liverpool. Br J Psychiatry 1992; 161: 230-239.

Copeland JRM, Dewey ME, Griffiths-Jones HM. Computerised psychiatric diagnosis system and case nomenclature for elderly subjects: GMS and AGECAT. Psychol Med 1986; 16: 89-99.

Copeland JRM, Dewey ME, Wood N, et al. Range of mental illness amongst the elderly in the community: prevalence in Liverpool using the GMS-AGECAT package. $\mathrm{Br} \mathrm{J}$ Psychiatry 1987; 150: 815-823.

Dartigues JF, Gagnon M, Michel P et al. The Paquid research program on the epidemiology of dementia. Methods and initial results. Rev Neurol 1991; 147 (3): 225-230.

Dawber TR, Kannel WB, Lyell LP. An approach to longitudinal studies in a community: The Framingham Study. Ann NY Acad Sci 1963; 107: 539-56.

Dewey ME, Copeland JRM, Lobo A et al. Computerised diagnosis from a standardised history schedule: a preliminary communication about the organic section of the HASAGECAT system. Int J Geriatr Psychiatry 1992; 7: 443-446.

Folstein MF, Folstein SE, McHugh PR. "Mini-Mental State". A practical method for grading the cognitive state of patients for the clinician. J Psychiatr Res 1975; 12 (3): 189-198.

Folstein MF, Spear S, Anthony JC et al. Dementia: Case ascertainment in a community survey. J Gerontology 1991 ; 46: 132-138.

Fratiglioni L, Launer LJ, Andersen K et al. Incidence of dementia and major subtypes in Europe: A collaborative study of population-based cohorts. Neurologic diseases in the elderly research group. Neurology 2000; 54(11 Suppl 5): S10-S15.

Hachinski VC, Iliff LD, Zilhka E et al. Cerebral blood flow in dementia. Arch Neurol 1975; 32(9): 632-637.

Hofman A, Rocca WA, Brayne C et al. The prevalence of dementia in Europe. A collaborative study of 1980-1990 prevalence findings. Int J Epidemiol 1991; 20(3): 736-748.

Instituto Nacional de Estadística. Movimiento natural de la población española, año 1987. Tomo I: resultados a nivel nacional y su distribución por provincias y capitales. Madrid: Ed. I.N.E. Artes Gráficas; 1992.

Kaelber CT, Regier DA. Directions in psychiatric epidemiology. Curr Opin Psychiatry 1995; 8(2): 109-115.

Katz S, Ford AB, Moskowitz RW et al. Studies of illness in the aged. The Index of ADL: A standardized measure of biological and psychosocial function. The J Am Med Assoc 1963; 185: 914-919.

Launer LJ, Brayne C, Dartigues JF et al. European studies on the incidence of dementing diseases. A Report of the EURODEM Research Group. Neuroepidemiology 1992; 211(suppl 1): 1-22.

Lawton MP, Brody EM. Assessment of older people: selfmaintaining and instrumental activities of daily living. Gerontologist 1969; 9(3): 179-86.

Letenneur L, Gilleron V, Commenges D et al. Are sex and educational level independent predictors of dementia and Alzheimer's disease? Incidence data from the PAQUID project. J Neurol Neurosurg Psychiatry 1999; 66(2): 177-183.

Lobo A, Escolar V et al. El "Mini-Examen Cognoscitivo": un test sencillo, práctico, para detectar alteraciones intelectivas en pacientes psiquiátricos. Rev Psiquiatr Psicol Med 1980; 14(5): 39-57.

Lobo A, Launer LJ, Fratiglioni L et al. Prevalence of dementia and major subtypes in Europe: A collaborative study of population-based cohorts. Neurology 2000; 54(Supl 5): 4-9.

Lobo A, Saz P, Día, JL et al. El Geriatric Mental State en poblaciones españolas: estudio de validación de parámetros "orgánicos" y "afectivos". Actas XIII Reunión Nacional de la Sociedad Española de Psiquiatría Biológica Zaragoza 1988; 333-340. 
Lobo A, Saz P, Día JL. The AGECAT “organic” section as a screening instrument for minor cognitive deficits. Psychiatr J Univ Ott 1990; 15(4): 212-215.

Lobo A, Saz P, Marcos G et al. The prevalence of dementia and depression in the elderly community in a Southern European population: the Zaragoza study. Arch Gen Psychiatry 1995; 52: 497-506.

Lobo A, Saz P, Marcos G et al. The Zaragoza Study: Dementia and depression in the elderly community. Barcelona: Masson Salvat S.A.; 1997.

Lobo A, Saz P, Marcos G et al. Adaptación española: Examen Cognoscitivo Mini Mental. Madrid: TEA Ediciones; 2002.

Lobo A, Saz P, Marcos G et al. The ZARADEMP Project on the incidence, prevalence and risk factors of dementia (and depression) in the elderly community: I. The context and the objectives. Eur J Psychiatry (in press) (this issue).

MacMahon B, Pugh TF. Epidemiology: Principles and Methods. Boston: Little, Brown; 1970.

McKahnn G, Drachman D, Folstein M et al. Clinical diagnosis of Alzheimer's disease: Report of the NINCDSADRDA Work Group under the auspices of Department of Health and Human Services Task Force on Alzheimer's Disease. Neurology 1984; 4: 939-944.

Riedel-Heller SG, Busse A, Aurich C et al. Incidence of dementia according to DSM-III-R and ICD-10: results of the Leipzig Longitudinal Study of the Aged (LEILA75+), Part. 2. Br J Psychiatry 2001; 179: 255-260.

Roman GC, Tatemichi TK, Erkinjuntti T et al. Vascular dementia: diagnostic criteria for research studies. Report of the NINDS-AIREN International Workshop. Neurology 1993; 43: 250-260.

Saz P, Día JL, De-la-Cámara C et al. Reliability and validity of the Spanish versión of the GMS-AGECAT package for the assessment of dementia and cognitive disturbances. Int $J$ Geriatr Psychiatry 1996; 11: 721-728.
Saz P. La batería GMS-AGECAT (Geriatric Mental StateAutomated Geriatric Examination for Computer Assisted Taxonomy) en la población geriátrica española: Validación y estudio de su utilidad en una muestra representativa de la comunidad. Tesis Doctoral. Universidad de Zaragoza; 1991.

Schlesselman JJ, Stolley PD. Case-control studies. Design, conduct, analysis. New York-Oxford: Oxford University Press; 1982. p. 144-170.

Steenland K, Henley J. Thun M. All-cause and cause-specific death rates by educational status for two million people in two American Cancer Society cohorts, 1959-1996. Am J Epidemiol 2002; 156 (1): 11-21.

Sulkava R, Wikström J, Aromaa A et al. Prevalence of severe dementia in Finland. Neurology, 1985; 35(7): 10251029.

Tárraga LL. Evaluación del deterioro cognitivo y funcional de la demencia. Escalas de mayor interés en la Atención Primaria. In:. Boada M, Tárraga LL, editors. El médico ante la demencia y su entorno, Módulo 1. Barcelona: Bayer S.A.; 1995. p. 37-50.

World Health Organization. Guidelines for the management of mild hypertension. Memorandum from a World Health Organization/International Society of Hypertension meeting. Guidelines Subcommittee of the WHO/ISH Mild Hypertension Liaison Committee. Hypertension 1993; 22(3): 392-403.

World Health Organization. The ICD-10 classification of mental and behavioral disorders: Clinical descriptions and diagnosis guidelines. Geneva: World Health Organization; 1992.

Address of correspondence:

Dr. Antonio Lobo

Servicio de Psiquiatría

Hospital Clínico Universitario, planta 3

Avda. San Juan Bosco, 15

50009 Zaragoza, Spain.

e-mail: alobo@unizar.es 